\title{
Ping Pong Cub
}

\author{
Gary Beaver ${ }^{1}$ \\ ${ }^{1}$ Affiliation not available
}

March 26, 2021

Grade: $5-7$

Time: roughly 50 minutes

\section{Goals:}

- Students will design and build a device that delivers a ping pong ball to a cup.

- Students are going to be able to explain design considerations based on kinetic energy, and forces

- Students will be able to finish the device by the end of class

\section{Vocabulary:}

- Elastic Potential Energy: Potential energy due to tension - either stretch

- Energy: The ability to do work. Appears in many forms, all of which are either kinetic or potential

- Force: A push or a pull. An influence on a body or system, causing or tending to cause a change in movement or shape.

- Friction: Forces resisting motion between one set of molecules and another due to electrical attraction and repulsion, usually between two solid surfaces; static before motion starts and kinetic during motion.

- Kinetic Energy (KE): Energy of motion. Includes heat, sound, and light (motion of molecules)

- Mass: The amount of matter that is contained by an object.

- Momentum: The quantity of motion of a moving object, equal to the product of its mass and its velocity.

\section{Materials per team (2-3)}

- $5 \mathrm{~cm}$ of Tape

- $30 \mathrm{~cm}$ of 3-ply string

- 4 Rubber Bands

- 1 Dixie Paper Cup

- 1 Sheet of Copy Paper

- 2 Paperclips

- Brown Paper Lunch Bag 


\section{Lesson Plan:}

\section{Introduction (3-5 minutes)}

1. The United States and its territories encompasses 169 geologically active volcanoes that are monitored by the United States Geological Survey (USGS). In order to prevent loss of life and property, the USGS issues volcano warnings which requires real-time monitoring of volcanoes, their seismology, and gas, thermal, and surface deformation measurements.

2. What challenges do scientists have to overcome in order to monitor volcanoes? What tools do you think scientists use in order to overcome these challenges? (Reference on Volcano Monitoring: http: //volcanoes.usgs.gov/about/faq/faqmonitoring.php)

3. Imagine if you had to deposit a small piece of equipment to the center of a lava flow. How would you do it? What science concepts would you use to help you achieve your mission?

\section{Starting Challenge (25 minutes)}

1. Introduce the Challenge: Devise a way to deposit a ping-pong ball into a paper cup that is located in the middle of a 6 -foot diameter circle.

2. Introduce the Constraints: Every person in the team must be actively involved in the placement of the ball. The ping pong ball must start outside the circle and must come to rest inside the paper cup in the center of the circle. Students may not touch the ping pong ball or reach into the 6-foot circle. No part of anyone's body may extend into the imaginary cylinder that extends above the circle. Only the provided materials may be used.

3. Build: Give students about 15 minutes to build and test. The instructor should ask open-ended questions to help guide students through the design process, but should also allow students space to tinker.

4. Testing: During the build time students should be able to test and iterate their designs freely. Students should build their device away from the testing site, and then bring their device over to the testing site in order to test. Teams may not build/iterate in at the test site! The setup for the test site is as follows:

- Create a 6 foot diameter circle out of tape, string, butcher paper, or a circular table cloth.

- Securely tape a cup (larger cups for an easier challenge, smaller cups for a more difficult challenge) to the center of the circle.

- Teams test from along the outer edge of the circle and may not extend any body part into the imaginary cylinder that extends above the circle.

\section{Demonstrating and Reflecting (20 minutes)}

1. Demonstrating: Have students demonstrate their devices one team at a time. If students have not completed their device, or their device did not function as expected, ask them how the device would have worked.

2. Reflecting: Have each group of students explain their design strategy and how their device uses energy and force to place the ball in the cup. The instructor should ask leading questions to get at the science behind the designs. 